\title{
Neuronal mechanisms of ginseng on antiobesity effects: implication of its synergistic benefits with physical exercise
}

\author{
Hyun Jung Park ${ }^{1, \dagger}$, Sung Ja Rhie ${ }^{2, \dagger}$, Insop Shimm ${ }^{3, *}$ \\ 'Department of Food Science and Biotechnology, Kyonggi University, Suwon, Korea \\ ${ }^{2}$ Department of Beauty Design, Halla University, Wonju, Korea \\ ${ }^{3}$ Department of Physiology, College of Medicine, Kyung Hee University, Seoul Korea
}

Obesity is a chronic disease of increasing prevalence in most countries, which leads to substantial increase in morbidity, and mortality in association with diabetes, hyperlipidaemia, hypertension, and other cardiovascular diseases. Many factors have been attributed to an epidemic of obesity including sedentary lifestyle, high-fat diets (HFD), and consumption of large amount of modern fast foods. Panax ginseng C. A. Meyer (PG) has several pharmacological and physiological effects. In particular, PG and saponin fractions from PG show a variety of efficacies such as antifatigue, hyperlipidemia, hypertension and noninsulin-dependent diabetes mellitus and obesity. We have revealed that ginseng and ginsenosides can decrease food intake energy expenditure by stimulating appetite regulatory hormones and can reduce energy intake. Exercise/ physical activity is well known as modality for treating the disease of overweight and obesity. It is suggested that natural products and their combinations with exercise may produce a synergistic activity that increases their bioavailability and action on multiple molecular targets, offering advantages over chemical treatments. This review is aimed at evaluating the antiobesity efficacy of ginseng and ginsenosides and delineating the mechanisms by which they function. Finally, we review information regarding interactions between ginseng and physical exercise in protecting against weight gain and obesity.

Keywords: Antiobesity, Ginseng, Leptin, Cholecystokinin, Neuropeptide Y, Exercise

\section{INTRODUCTION}

Most people agree that physical activity is an essential component to maintaining a healthy lifestyle. Epidemiological evidence has shown a positive relationship between obesity and dietary fat intake. Many studies reported that rats and mice prove a similar relationship, and they showed an appropriate model for dietary obesity studying (Park et al., 2019; van der Klaauw, 2018). Overweight and obesity are related as excessive fat accumulation or abnormal that presents a risk to health. An overview of animal studies had shown that increased fat intake is associated with metabolic disease and cardiovascular-related diseases. Obesity is defined medically as a state of increased body weight (Spiegelman and Flier, 2001) and is associated with several medical problems such as cardiovascular disease, type 2 diabetes, and several cancers (Haslam and James, 2005). Body is need for energy which is obtained from food. If energy intake exceeds total body energy expenditure, stored energy increases. Thus, increased food intake explains the increase in body weight vice versa. During the last few decades, there is a need for better tolerated antiobesity drugs expanding the understanding of peripheral signals and central nervous system pathways involved in the regulation of adiposity to treat obesity. Many therapeutic herbs and nutrients have far fewer side effects and may provide an alternative treatment or could be used to enhance the effect of prescription medications.

It has been demonstrated that saponins from Platycodi radix,
${ }^{*}$ Corresponding author: Insop Shim (D https://orcid.org/0000-0002-8014-9782 Department of Physiology, College of Medicine Kyung Hee University,

26 Kyungheedae-ro, Dongdaemun-gu, Seoul 02447, Korea

Email: ishim@khu.ac.kr

${ }^{\dagger}$ These authors contributed equally to this study as co-first authors.

Received: October 22, 2021 / Accepted: November 30, 2021
This is an Open Access article distributed under the terms of the Creative Commons Attribution Non-Commercial License (https://creativecommons.org/licenses/by-nc/4.0/) which permits unrestricted non-commercial use, distribution, and reproduction in any medium, provided the original work is properly cited. 
herbal supplement containing Ephedrae herba, dietary tea saponin, and ginseng berry extract, have antiobesity or antidiabetic actions (Han et al., 2001; Han et al., 2002). Korean red ginseng (KRG, Ginseng Radix Rubra), which is one of several types of Panax ginseng $C$. A. Meyer (PG), has several pharmacological and physiological effects that are being gradually disclosed. In particular, KRG or its saponin fractions show a variety of efficacies such as anticancer, antihypertension, antidiabetes, antinociception, and improving weak body conditions as tonics (Choi, 2008).

Previous studies demonstrated that the crude saponin (CS) of KRG had an antiobesity effect (Kim et al., 2005; Park et al., 2019). Ginseng CS is known to contain a variety of bioactive saponins. Some pharmacological effects of ginseng were verified as being due to different types of ginsenosides. For example, the protopanaxadiol (PD)-type ginsenosides Rb1, Rb2, Rc, and Rd have an antioxidant activity, whereas the protopanaxatriol (PT)-type ginsenosides Re, Rf and $\mathrm{Rg} 1$ have efficacy on the improvement of learning and memory (Jaenicke et al., 1991). The antiobesity activity of PD- and PT-type saponins, major active compounds isolated from CS has been reported (Kim et al., 2005). Furthermore, we have demonstrated that several ginsenosides, Rb1, Rd, Rg1, and Re reduced HFD-induced obesity. Persuasive evidence exists to indicate that gastrointestinal factors, cholecystokinin (CCK), and neuropeptide Y (NPY) release provide feedback that slows gastric empty and regulates energy intake in response to combinations of intradermal fat and or carbohydrate (Luscombe-Marsh et al., 2013; Montgomery et al., 2013).

Other studies have shown that ginseng root extract significantly decreased body weight and adipose tissue mass in other animal models of obesity, such as Otsuka Long-Evans Tokushima fatty rats, oblob mice, and HFD-fed Sprague-Dawley rats (Lee et al., 2012; Mollah et al., 2009). In addition, Lee et al. (2013) demonstrated that KRG decreases fat mass and inhibits obesity in HFDfed obese C57BL/6J mice. Based on ability of KRG to decrease body weight and adipocyte size and also, KRG inhibits obesity by regulating the expression of adipogenesis-associated genes.

Exercise/physical activity is well known as modality for treating the disease of overweight and obesity. It is suggested that natural products and their combinations with exercise may produce a synergistic activity that increases their bioavailability and action on multiple molecular targets, offering advantages over chemical treatments. This review is aimed at evaluating the antiobesity efficacy of ginseng and ginsenosides and delineating the mechanisms by which they function. Finally, we review information regarding interactions between ginseng and physical exercise in protecting against weight gain and obesity.

\section{EFFECT OF CRUDE SAPONIN KRG ON HIGH-FAT DIET-INDUCED OBESITY IN THE RAT}

Several studies have shown that saponins from natural product have an antiobesity effect. CS isolated from Platycodi radix prevented increases in the body weight, adipose tissue weight, and liver triacylglycerol in mice fed HFD (Han et al., 2002; Kim et al., 2009). Diet is a major factor within the current obesogenic environment, where energy dense, high fat, high sugar foods and beverages engage both homeostatic and hedonic regulatory systems. A number of different types of diet-induced obesity model have been employed to induce obesity and research characterizing the outbred Sprague-Dawley rat has frequently employed a high energy diet (Levin and Dunn-Meynell, 1997; Levin and Keesey, 1998). The Sprague-Dawley rat represents a useful model of obesity on a Western diet. Phenotypic variation in HFD-induced obesity within inbred strains of mice was observed by gene expression analyses. The over- or underexpression of selective genes before the HFD was correlated with adiposity (Koza et al., 2006).

We previously reported the antiobesity effects of CS of KRG in the rat fed a HF diet study (Kim et al., 2005). The antiobesity effects of CS were investigated in rats fed a normal or HFD by measuring changes in body weight, food consumption, serum level of leptin, and neuronal response of NPY in the hypothalamus. Male Sprague-Dawley rats became obese by feeding the HF diet over 5 weeks, while the control rats were fed a normal diet, and then both groups were treated with CS (200 mg/kg, intraperitoneally) for 3 weeks. The body weight, food consumption, adipose tissues, and expression of appetite peptides such as leptin and NPY were investigated in rats fed normal and HFD after treatment of CS. Administration of CS reduced body weight, food intake, and fat content in HFD rats in a manner similar to those of the normal diet-fed rats. The hypothalamic NPY expression and serum leptin level were reduced in HFD rats after CS treatment. These studies demonstrated that CS may be useful in the treatment of obesity and related disorders as antiobesity agents.

Tea saponin suppressed increases in body, parametrial adipose tissue weights, and diameter in adipose cell size induced by a HFD through delaying the intestinal absorption of dietary fat by inhibiting pancreatic lipase activity (Han et al., 2001). Administration of NPY in rats produced a substantial increase in body weight consisting primarily of increased fat mass (Zarjevski et al., 1993). The hypothalamus is well recognized for its importance in regulating sympathetic activity and energy balance. A few discrete nu- 
clei in the basal hypothalamus are crucial in the regulation of daily energy homeostasis, especially those sites connected with neural mechanisms affecting appetite. The inference that hypothalamic sites, such as the arcuate nucleus (ARC), lateral hypothalamus (LH), ventromedial hypothalamus (VMH), paraventricular nucleus (PVN), and other nuclei, contain neural mechanisms affecting eating behavior was based on the results of numerous studies employing either discrete lesion in the hypothalamus or surgical transection of neural pathways (Sclafani, 1971). This report suggests that the anorexigenic effect of CS in HFD-induced obese rats may be, in part, mediated through the NPY ARC-PVN projection.

CS of KRG has been shown to exert antiobesity effects in rats fed with HF diet by reducing of body weight, food consumption, and fat storage. CS of KRG is also effective in the regulation of serum leptin and NPY expression in the hypothalamus when obesity is induced with a HFD. These results suggest the possibility that the action of CS of KRG is related to the suppression of the desire for food.

It is known that CS contained nine major ginsenosides and other minor ginsenosides and components (Zarjevski et al., 1993), suggesting that these ginsenosides or their components may contribute to the antiobesity activities of ginseng CS.

\section{ANTIOBESITY EFFECTS OF THE PROTOPANAXADIOL-AND PROTOPANAXATRIOL-TYPE SAPONINS OF RED GINSENG}

Ginseng CS contain a variety of bioactive saponins, including PD- and PT-type saponins, major active compounds isolated from CS. In our studies, it was demonstrated that two major red ginseng saponins, PD- and PT-type saponins, reduced the HFD-induced obesity (Kim al., 2009). Treatment with PD and PT in the HFD group reduced the body weight, total food intake, fat contents, serum total cholesterol and leptin to levels equal to or below the $\mathrm{N}$ diet group. The hypothalamic expression of orexigenic NPY was significantly decreased with PD or PT treatment, whereas that of anorexigenic cholecystokinin was increased, compared with the control HFD group. In addition, PD-type saponins had more potent antiobesity properties than PT saponins, indicating that PDtype saponins are the major components contributing to the antiobesity activities of ginseng CS. The results suggest that the antiobesity activity of PD- and PT-type saponins may result from inhibiting energy gain, normalizing hypothalamic neuropeptides and serum biochemicals related to the control of obesity.
A previous study reported that the administration of CS from red ginseng (200 mg/kg, intraperitoneally) for 3 weeks reduced the weight of the body, the parametrical adipose tissues and the levels of serum lipid and leptin in the HFD group (Kim et al., 2005). It seems that the antiobese effects of CS are due to the action of active compounds. Both the PD- and PT-type saponins, the representative compounds of $R G$, reduced the body weight, the body fats and the serum lipid and leptin levels in the HFD-induced obesity rats. Specially, the results of this study are of worth in terms of the fact that visceral adipose tissue was decreased after treatment with the PD-type saponin. Also, PD-type saponin also reduced the NPY-immunoreactive neurons of the LH and PVN, and it increased the CCK-immunoreactive neurons of the PVN, compared with the HFD group, but the PT-type saponins reduced the CCK-immunoreactive neurons of the VMH. These results suggested that PD was more effective than PT for the antiobesity effects related to the above factors. The reduced NPY immunoreactivity in the LH and PVN, but not the ARC, after treatment with PD-type saponins supported a result that was similar to our previous study (Kim et al., 2005); that is, PD-type saponins may inhibit the release of NPY or its transport to the PVN, rather than NPY synthesis in the ARC.

Taken together, PD- and PT-type saponins from RG have been shown to exert antiobesity effects in the rats fed with a HF diet by reducing their body weight their food consumption and their fat storage. They were also effective in the regulation of serum lipid and leptin and the hypothalamic NPY and CCK expressions. In addition, PD-type saponins have more potent antiobesity properties than PT-type saponins for the factors related to HFD-induced obesity, indicating that PD-type saponins are the major component contributing to the antiobesity activities of ginseng CS.

\section{ANTIOBESITY EFFECTS OF GINSENOSIDES IN HIGH-FAT DIET-FED RATS}

Since PD- and PT-type saponins from RG were shown to produce antiobesity effects, the antiobesity effects of several single ginsenosides in KRG have been studied. Treatment of Rd, Re, and Rb1 markedly decreased body fat mass and body weight (Park et al., 2019). Ginsenosides (Rb1, Rd, Rg1, and Re) reduced HFDinduced obesity. Body weight, total food intake, and serum leptin were reduced in the groups treated with Rb1, Re, Rd, Rg1 and total regional fat storage was reduced in the group treated with $\mathrm{Rb} 1, \mathrm{Rd}, \mathrm{Re}, \mathrm{Rg} 1$ in order. Serum nitric oxide (NO) was reduced in the groups treated with Re, Rd, Rb1, Rg1, in that order. In 
addition, Rb1 reduced NPY-immunoreactive neurons of the PVN and increased CCK-immunoreactive neurons of the PVN. Rb1 tended to increase physical activity in the early period after treatment. These results suggest that $\mathrm{Rb1}$ may have the most effective antiobesity activity.

PD-type ginsenoside Rb1 from the CS of KRG may be a useful compound for the treatment of obesity and related disorders through the modulation of peripheral and central appetite-regulating signals. The HFD ( $40 \%$ of calories as fat) used in this study induced obesity through increased body weight and fat accumulation. Although cumulative food intake over the experimental period was similar in both groups, the HFD resulted a marked increase in body weight and body fat mass after 8 weeks relative to the $\mathrm{N}$ diet group. These results were similar to those of our previous study. However, the treatment of ginsenosides (Rb1, Re, Re) normalized the body weight and the fat mass in the rats. Previously, it was reported that the ginsenoside $\mathrm{Rb} 1$ reduced fatty liver through the activation of adenosine monophosphate-activated protein kinase (AMPK) in obese rats (Shen et al., 2013). The activation of AMPK indicated an increase in ATP usage. Xiong et al. (2010) reported the antiobesity and antihyperglycemic effect of ginsenoside $\mathrm{Rb} 1$ in rats. Collectively, these data suggest that the ginsenoside $\mathrm{Rb} 1$ may be helpful in the prevention of diet-induced obesity and metabolic syndrome.

Also, food intake is regulated through the central nervous system pathway, mediated by various neurotransmitters including NPY and CCK. Hypothalamic NPY potentially stimulates food intake (Fan et al., 2016) and CCK has been reported to reduce appetite and inhibits food intake. Add Reference. In this study, there were few differences between the high fat and normal diets with respect to the hypothalamic NPY and CCK expression, but there was a tendency for lower NPY levels and higher CCK levels in the HFD group through diet composition, leptin, and NO level. In this study, it is more likely that decreased serum leptin and $\mathrm{NO}$ after the treatment of ginsenoside $\mathrm{Rb} 1$ resulted from decreased body fat. In contrast, leptin acts partly through interactions with NPY in the hypothalamus (Lin et al., 2000). The lower NPY level in the hypothalamus of the HFD group than the normal diet group was closely associated with an increase in circulating leptin levels. The decreased expression of NPY in the HFD group after treatment with ginsenoside $\mathrm{Rb1}$ may also reflect the reduced leptin levels related to body fat mass.

Also, food intake is regulated through the central nervous system pathway, mediated by various neurotransmitters including NPY and CCK. Hypothalamic NPY potentially stimulates food intake (Fan et al., 2016) and CCK has been reported to reduce appetite and inhibits food intake. As leptin is known to act on the ARC-PVN feeding regulatory pathway, the inhibition of the signal that produces a balance involving NPY arcuato-paraventricular projection by leptin may be one of the potential mechanisms underlying antiobesity action of ginsenoside Rb1 (Kalra and Kalra, 2003). There were few differences between the HF and normal diets with respect to the hypothalamic NPY and CCK expression, but there was a tendency for lower NPY levels and higher CCK levels in the HFD group through diet composition, leptin, and NO level. It is more likely that decreased serum leptin and NO after the treatment of ginsenoside Rb1 resulted from decreased body fat. In contrast, leptin acts partly through interactions with NPY in the hypothalamus (Lin et al., 2000). The lower NPY level in the hypothalamus of the HFD group than the normal diet group was closely associated with an increase in circulating leptin levels. The decreased expression of NPY in the HFD group after treatment with ginsenoside $\mathrm{Rb} 1$ may also reflect the reduced leptin levels related to body fat mass. Furthermore, the ARC contains a high density of neurons that produce the orexigenic and anorexigenic peptides and the terminal fields of neurons in the ARC extend into various hypothalamic sites, such as $\mathrm{LH}, \mathrm{VMH}$, and PVN (Bischof and Park, 2015; Grundmann et al., 2005). In the HFDfed rats, increased CCK-immunoreactive neurons of the ARC, $\mathrm{VMH}$, and PVN were shown. The treatment of ginsenoside Rb1 significantly increased the CCK-immunoreactive neurons in the VMH and PVN. Consistent with behaviors as food intake and locomotor activity, the treatment of ginsenoside Rb1 acts as an obesity-related neuromodulator.

In conclusion, ginsenoside $\mathrm{Rb} 1$ was found to exert antiobesity effects in the rats fed HFD through the reduction of their body weight, food intake, and fat storage. It was also effective in the regulation of serum leptin and NO secretion, and CCK and NPY expression in the hypothalamus. Rb1 may be a useful agent for the control of obesity via the peripheral and central appetite modulators.

\section{EFFECT OF COMBINATION TREATMENT OF PG OR ITS COMPOUNDS WITH EXERCISE}

Obesity represents a significant public health concern with onethird of adults classified as living with obesity in the United States. It correlates with cardio-metabolic comorbidities that can decrease the quality of life. PG is medicinal herb which has been used for over 2,000 years in Asia. Also, PG has been used for benefiting Qi 
for thousand years. Researchers have proposed that exercise is an important lifestyle measure to maintain a healthy weight. This part of review covers the role of exercise in obesity and fitness.

An enhancing effect of black ginseng extract (BGE) was reported on exercise capacity in an endurance exercising animal model. It was shown that when experimental animals were treated with BGE and their exercise ability in the maximal running distance test was examined using a treadmill every week for 6 weeks, the exercise capacity of 150 and $300 \mathrm{mg} / \mathrm{kg}$ administrated group was significantly increased, and blood lactic acid level was decreased. BGE is known to contain a large amount of ginsenoside Rh, Rg2, and $\mathrm{Rg} 3$, which reported higher effects such as anti-inflammatory, anticancer, antistress, and antioxidant than red ginseng in humans and animals (Leung et al., 2007). In addition, recent studies have reported that administration of ginseng can increase maximum oxygen intake and long-term exhaustion exercise, decrease heart rate during rest, and increase arteriovenous blood oxygen intersection (Bucci, 2000). It is believed that the administration of BGE increased the activity of CS in the muscles, resulting in increased muscle oxidation and respiratory capacity (Fu and Ji, 2003). In general, exercise-related muscle metabolism undergoes major changes that increase oxidation power and delay muscle fatigue conditions. Therefore, it seems that BGE administration along with regular exercise significantly reduces serum lactic acid concentration and increases maximum exercise distance due to increased muscle oxidation and respiratory capacity due to increased CS activity in muscles. These results also suggested that BGE can be used as a candidate supplement of health food products for promoting endurance exercise capacity in human athletes.

In human study, PG extract intake influenced exercise-induced muscle damage and inflammation responses. Eighteen male college students were randomly assigned to either an RG intake group or a placebo group. All subjects performed a high-intensity uphill treadmill running task (two rounds of $45 \mathrm{~min}$ at $10 \mathrm{~km} / \mathrm{hr}$ speed with 150 uphill slope separated by $5 \mathrm{~min}$ of rest). The RG group ingested $20 \mathrm{~g}$ /day of KRG extract (mixed with $200 \mathrm{~mL}$ of water) 3 times/day for 7 days prior to performing the uphill treadmill exercise test and for 4 days after the treadmill test, while the $\mathrm{P}$ group ingested $200 \mathrm{~mL}$ of water containing Agastachis Herba on the same schedule (Jung et al., 2011). Plasma creatine kinase activity (CK) and interleukin (IL)-6 levels were measured at pre-exercise and 24-, 48-, 72-, and 96-hr postexercise; the IL-6 level was also measured at 1- and 2-hr postexercise. To evaluate insulin sensitivity, the oral glucose tolerance test was performed 24-hr postexercise. Plasma CK level in RG was significantly lower than that in
P 72-hr postexercise, and IL-6 level was significantly decreased in RG during the 2-and 3-hr recovery period compared to that of $P$. Plasma glucose and insulin responses in RG were significantly reduced compared to those of $\mathrm{P}$.

IL-6 is a pleiotropic cytokine that has important roles in the regulation of the immune response, inflammation, and hematopoiesis (Nishimoto and Kishimoto, 2006). It has drawn a lot of attention from researchers in recent years since it is markedly increased after exercise, a response that is considered to be associated with inflammation (Fischer et al., 2007). Also, the reason for this improvement in insulin sensitivity may be associated with reduced muscle damage resulting from RG intake. Many studies have suggested that muscle damage due to strenuous muscle contraction may reduce insulin sensitivity in humans and rats (Ide et al., 1996; King et al., 1993), while others have found some indications that ginseng administration improves insulin sensitivity in humans and rats (Lin et al., 2008). In the human study, one possible mechanism of exercise-induced muscle damage reduction due to RG supplementation might be associated with the anti-oxidative effects of ginseng. Some studies reported the intense bouts of exercise induce oxidative stress, suggesting that muscle tissue is a target for damage via free radical medicated mechanisms (Hsu et al., 2005; Kendall and Eston, 2002). Also, high intensity exercise may result in a markedly enhanced an attack of free radicals on the cell membranes may lead to a decrease in cell viability. The results of this study suggest that RG supplementation could reduce exercise-induced muscle damage and inflammatory responses, resulting in improvements in insulin sensitivity

\section{CONCLUSIONS}

Ginseng or ginsenosides may help control appetite and prevent the over-intake of food energy by attenuating the HFD-induced chronic inflammation of the hypothalamus, improving leptin resistance, and reducing the secretion of NPY. CS of KRG has been shown to exert antiobesity effects in rats fed with HFD by reducing of body weight, food consumption, and fat storage. CS of KRG is also effective in the regulation of serum leptin and NPY expression in the hypothalamus when obesity is induced with a HFD. Also, ginsenosides, PD- and PT-type saponins from RG have been shown to exert antiobesity effects in the rats fed with a HFD by reducing their body weight their food consumption and their fat storage. They were also effective in the regulation of serum lipid and leptin and the hypothalamic NPY and CCK expressions. Furthermore, ginsenoside Rb1 was found to exert antiobesity effects 


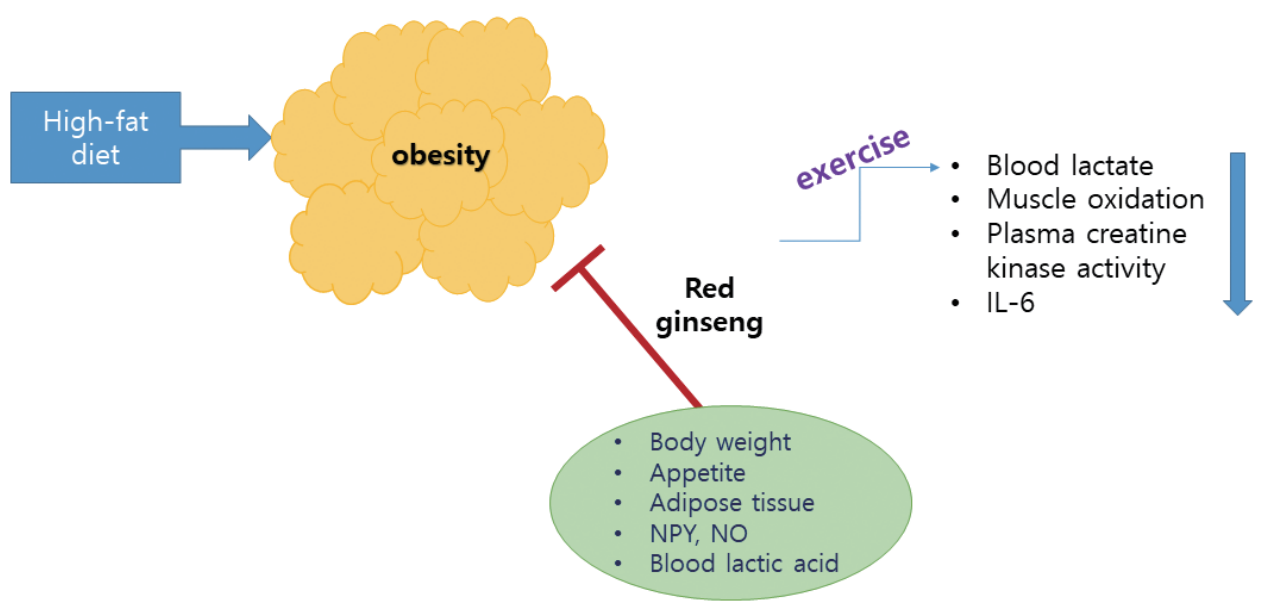

Fig. 1. Action mechanisms of red ginseng with exercise. IL-6, interleukin-6; NPY, neuropeptide Y; NO, nitric oxide.

in the rats fed HFD through the reduction of their body weight, food intake, and fat storage. It was also effective in the regulation of serum leptin and NO secretion, and CCK and NPY expression in the hypothalamus.

These results suggest the possibility that the action of CS of $\mathrm{KRG}$ is related to the suppression of the desire for food. In addition, PD-type saponins have more potent antiobesity properties than PT-type saponins for the factors related to HFD-induced obesity, indicating that PD-type saponins are the major component contributing to the antiobesity activities of ginseng CS. Rb1 may be a useful agent for the control of obesity via the peripheral and central appetite modulators There is limited evidence supporting the suggestion that ginseng showed an antiobesity effect in humans. Additional study and verification through longitudinal human studies are required to elucidate the antiobesity effects of ginseng in humans. Overall view of action mechanisms of red ginseng with exercise is summarized in the Fig. 1.

In conclusion, these results indicate that BGE have a potential for promoting exercise capacity by increasing CS activity in the muscle of rats. In human study, these results also suggested that BGE can be used as a candidate supplement of health food products for promoting endurance exercise capacity in human athletes. The results of this study suggest that RG supplementation could reduce exercise-induced muscle damage and inflammatory responses, resulting in improvements in insulin sensitivity.

\section{CONFLICT OF INTEREST}

No potential conflict of interest relevant to this article was reported.

\section{ACKNOWLEDGEMENTS}

This work was supported by grants from the National Research Foundation of the Korean government (NRF-NRF 2021R1A2C 1093825).

\section{REFERENCES}

Bischof GN, Park DC. Obesity and aging: consequences for cognition, brain structure, and brain function. Psychosom Med 2015;77:697-709.

Bucci LR. Selected herbals and human exercise performance. Am J Clin Nutr 2000;72(2 Suppl):624S-636S.

Choi KT. Botanical characteristics, pharmacological effects and medicinal components of Korean Panax ginseng C A Meyer. Acta Pharmacol Sin 2008;29:1109-1118.

Fan S, Dakshinamoorthy J, Kim ER, Xu Y, Huang C, Tong Q. An indirect action contributes to c-Fos induction in paraventricular hypothalamic nucleus by neuropeptide Y. Sci Rep 2016;6:19980.

Fischer CP, Berntsen A, Perstrup LB, Eskildsen P, Pedersen BK. Plasma levels of interleukin-6 and C-reactive protein are associated with physical inactivity independent of obesity. Scand J Med Sci Sports 2007;17: 580-587.

Fu Y, Ji LL. Chronic ginseng consumption attenuates age-associated oxidative stress in rats. J Nutr 2003;133:3603-3609.

Grundmann SJ, Pankey EA, Cook MM, Wood AL, Rollins BL, King BM. Combination unilateral amygdaloid and ventromedial hypothalamic lesions: evidence for a feeding pathway. Am J Physiol Regul Integr Comp Physiol 2005;288: R702-707.

Han LK, Kimura Y, Kawashima M, Takaku T, Taniyama T, Hayashi T, Zheng YN, Okuda H. Anti-obesity effects in rodents of dietary teasa- 
ponin, a lipase inhibitor. Int J Obes Relat Metab Disord 2001;25:14591464.

Han LK, Zheng YN, Xu BJ, Okuda H, Kimura Y. Saponins from platycodi radix ameliorate high fat diet-induced obesity in mice. J Nutr 2002; 132:2241-2245.

Haslam DW, James WP. Obesity. Lancet 2005;366:1197-1209.

Hsu CC, Ho MC, Lin LC, Su B, Hsu MC. American ginseng supplementation attenuates creatine kinase level induced by submaximal exercise in human beings. World J Gastroenterol 2005;11:5327-5331.

Ide K, Higaki Y, Nishizumi M, Kiyonaga A, Shindo M, Tanaka H. Impaired non-insulin mediated glucose uptake after downhill running in rats. Life Sci 1996;59:1601-1605.

Jaenicke B, Kim EJ, Ahn JW, Lee HS. Effect of Panax ginseng extract on passive avoidance retention in old rats. Arch Pharm Res 1991;14:25-29.

Jung HL, Kwak HE, Kim SS, Kim YC, Lee CD, Byurn HK, Kang HY. Effects of Panax ginseng supplementation on muscle damage and inflammation after uphill treadmill running in humans. Am J Chin Med 2011;39:441-450.

Kalra SP, Kalra PS. Neuropeptide Y: a physiological orexigen modulated by the feedback action of ghrelin and leptin. Endocrine 2003;22:49-56.

Kendall B, Eston R. Exercise-induced muscle damage and the potential protective role of estrogen. Sports Med 2002;32:103-123.

Kim JH, Hahm DH, Yang DC, Kim JH, Lee HJ, Shim I. Effect of crude saponin of Korean red ginseng on high-fat diet-induced obesity in the rat. J Pharmacol Sci 2005;97:124-131.

Kim JH, Kang SA, Han SM, Shim I. Comparison of the antiobesity effects of the protopanaxadiol- and protopanaxatriol-type saponins of red ginseng. Phytother Res 2009;23:78-85.

King DS, Feltmeyer TL, Baldus PJ, Sharp RL, Nespor J. Effects of eccentric exercise on insulin secretion and action in humans. J Appl Physiol (1985) 1993;75:2151-2156

Koza RA, Nikonova L, Hogan J, Rim JS, Mendoza T, Faulk C, Skaf J, Kozak LP. Changes in gene expression foreshadow diet-induced obesity in genetically identical mice. PLoS Genet 2006;2:e81.

Lee H, Park D, Yoon M. Korean red ginseng (Panax ginseng) prevents obesity by inhibiting angiogenesis in high fat diet-induced obese C57BL/6 mice. Food Chem Toxicol 2013;53:402-408.

Lee SH, Lee HJ, Lee YH, Lee BW, Cha BS, Kang ES, Ahn CW, Park JS, Kim HJ, Lee EY, Lee HC. Korean red ginseng (Panax ginseng) improves insulin sensitivity in high fat fed Sprague-Dawley rats. Phytother Res 2012;26:142-147.

Leung KW, Yung KK, Mak NK, Chan YS, Fan TP, Wong RN. Neuroprotective effects of ginsenoside-Rg1 in primary nigral neurons against rotenone toxicity. Neuropharmacology 2007;52:827-835.

Levin BE, Dunn-Meynell AA. Dysregulation of arcuate nucleus prepro- neuropeptide Y mRNA in diet-induced obese rats. Am J Physiol 1997; 272(5 Pt 2):R1365-1370.

Levin BE, Keesey RE. Defense of differing body weight set points in diet-induced obese and resistant rats. Am J Physiol 1998;274:R412-419.

Lin E, Wang Y, Mehendale S, Sun S, Wang CZ, Xie JT, Aung HH, Yuan CS. Antioxidant protection by American ginseng in pancreatic $\beta$-cells. Am J Chin Med 2008;36:981-988.

Lin S, Storlien LH, Huang XF. Leptin receptor, NPY, POMC mRNA expression in the diet-induced obese mouse brain. Brain Res 2000;875: 89-95.

Luscombe-Marsh ND, Seimon RV, Bollmeyer E, Wishart JM, Wittert GA, Horowitz M, Bellon M, Feinle-Bisset C. Acute effects of oral preloads with increasing energy density on gastric emptying, gut hormone release, thermogenesis and energy intake, in overweight and obese men. Asia Pac J Clin Nutr 2013;22:380-390.

Mollah ML, Kim GS, Moon HK, Chung SK, Cheon YP, Kim JK, Kim KS. Antiobesity effects of wild ginseng (Panax ginseng C.A. Meyer) mediated by PPAR- $\gamma$, GLUT4 and LPL in ob/ob mice. Phytother Res 2009; 23:220-225

Montgomery IA, Irwin N, Flatt PR. Beneficial effects of (pGlu-Gln)-CCK-8 on energy intake and metabolism in high fat fed mice are associated with alterations of hypothalamic gene expression. Horm Metab Res 2013;45:471-473.

Nishimoto N, Kishimoto T. Interleukin 6: from bench to bedside. Nat Clin Pract Rheumatol 2006;2:619-626.

Park HJ, Kim JH, Shim I. Anti-obesity effects of ginsenosides in high-fat diet-fed rats. Chin J Integr Med 2019;25:895-901.

Sclafani A. Neural pathways involved in the ventromedial hypothalamic lesion syndrome in the rat. J Comp Physiol Psychol 1971;77:70-96.

Shen L, Xiong Y, Wang DQ, Howles P, Basford JE, Wang J, Xiong YQ, Hui DY, Woods SC, Liu M. Ginsenoside Rb1 reduces fatty liver by activating AMP-activated protein kinase in obese rats. J Lipid Res 2013;54: 1430-1438.

Spiegelman BM, Flier JS. Obesity and the regulation of energy balance. Cell 2001;104:531-543.

van der Klaauw AA. Neuropeptides in obesity and metabolic disease. Clin Chem 2018;64:173-182.

Xiong Y, Shen L, Liu KJ, Tso P, Xiong Y, Wang G, Woods SC, Liu M. Antiobesity and antihyperglycemic effects of ginsenoside $\mathrm{Rb} 1$ in rats. Diabetes 2010;59:2505-2512.

Zarjevski N, Cusin I, Vettor R, Rohner-Jeanrenaud F, Jeanrenaud B. Chronic intracerebroventricular neuropeptide- $Y$ administration to normal rats mimics hormonal and metabolic changes of obesity. Endocrinology 1993;133:1753-1758. 